\title{
Can unconscious sequential integration of semantic information occur when the prime Chinese characters are displayed from left to right?
}

\author{
Shen $\mathrm{Tu}^{1}$ (D) S Simin $\mathrm{Wan}^{2} \cdot$ Jerwen Jou ${ }^{3} \cdot$ Yidan $\mathrm{Ma}^{4} \cdot$ Guang Zhao ${ }^{5} \cdot$ Weigang Pan $^{6}$ \\ Published online: 16 July 2019 \\ (C) The Psychonomic Society, Inc. 2019
}

\begin{abstract}
Recent studies have investigated whether conscious awareness is necessary for semantic integration. Although results have varied, simultaneous presentation of words have consistently led to greater semantic integration than sequential presentation in a single location. The current studies were designed to investigate whether the disadvantage of sequential presentation for unconscious semantic integration is specific to unfamiliar word-by-word presentation in one location or extends to the more natural reading conditions of viewing items sequentially from left to right. In Experiment 1, when the first three characters of Chinese idioms were presented simultaneously under masked conditions, performance on a separate two-alternative forcedchoice recognition task was at chance level. Despite being unaware of the identity of prime characters, participants were faster to indicate that a subsequent item was a Chinese character when it was congruent with the beginning of the idiom, thus providing evidence of semantic integration. In contrast, when the three (Experiment 2) or two (Experiment 3) prime characters were presented sequentially in time from left to right, there was no evidence of semantic integration. These results indicate that unconscious semantic integration is more limited than previously reported, and may require simultaneous visual presentation.
\end{abstract}

Keywords Unconscious priming $\cdot$ Sequential integration $\cdot$ Semantic integration

Shen Tu and Simin Wan contributed equally to this work.

Electronic supplementary material The online version of this article (https://doi.org/10.3758/s13414-019-01816-2) contains supplementary material, which is available to authorized users.

Shen $\mathrm{Tu}$

shentu614@vip.163.com

1 Department of Psychology, School of Public Administration, Guizhou University of Finance and Economics, Guiyang 550025, China

2 Department of Psychology, Institute of Education, China West Normal University, Nanchong 637002, China

3 Department of Psychological Science, University of Texas-Rio Grande Valley, Edinburg, TX 78539, USA

4 Department of Psychology, Institute of Education Science, Leshan Normal University, Leshan 614000, China

5 School of Psychology, Liaoning Normal University, Dalian 116029, China

6 Laborartory of Emotion and Mental Health, Chongqing University of Arts and Sciences, Yongchuan 402160, China
In recent years, a few studies have investigated unconscious semantic integration of multiple words, but these studies have yielded mixed results. Some studies have shown that semantic integration of multiple words can occur unconsciously. For example, using the continuous flash suppression technique, Sklar et al. (2012) demonstrated that the meaning of multiple words presented simultaneously could be integrated unconsciously. A similar result was observed using the backward masking technique and modifier-adjective pairs (e.g. "very/ good/peace"; "not/bad/murder") when the paired words were presented simultaneously (van Gaal et al., 2014). However, van Gaal et al. (2014) also found that conscious processing was necessary for semantic integration when the words were presented sequentially (van Gaal et al., 2014). These findings seem to suggest that the simultaneous presentation of prime words is necessary for unconscious semantic integration to occur.

In the present studies, we investigated unconscious sequential semantic integration using Chinese four-character idioms with a methodological change (i.e. the prime characters were spaced out from left to right rather than superimposing one on another) intended to facilitate possible semantic integration across characters. In Chinese, four-character idioms are 
commonly used as a single idiomatic expression. Chinese four-character idioms have a fixed structure and a fixed meaning, which cannot be derived from the individual meanings of the four characters literally. They are similar to a miniature sentence and represent a story or an idea. Most of them have metaphoric meaning besides their literal meaning. For example, the literal meaning of “废寝忘食” is “miss meals and sleep," but its idiomatic meaning is making extremely concentrated effort; similarly, the literal meaning of “月明星稀” is “the moon is bright and the stars are few." Its metaphoric meaning is that one prominent thing can eclipse other things around it. There are many reasons for using four-character idioms as experimental materials: high familiarity, fixed structures, and complex holistic meaning (a metaphoric, conventional meaning beyond the sum of the literal meanings of the characters). Therefore, there are obvious advantages to using Chinese four-character idioms to investigate the limitation of unconscious semantic integration.

Two previous studies have used Chinese four-character idioms as experimental materials to study unconscious semantic integration. The results showed that meaning integration could not occur without consciousness when the priming characters were presented sequentially using the crowded technique (Zhou, Lee, Li, Tien, \& Yeh, 2016) and using the continuous flash suppression technique (Yang, Tien, Yang, \& Yeh, 2017). We have two hypotheses as to why no semantic integration occurred in the above studies. One attributes the failure to the time intervals separating the priming characters, and the other to the spatial overlapping of the priming characters (the characters were displayed at the same spatial location) by which the multiple stimuli could fuse into a new stimulus (Eriksen, 1980; Felsten \& Wasserman, 1980) and produce some neural activity that hampers the unconscious integration of information (Andersen, Essick, \& Siegel, 1985).

Previous evidence showed that time interval played an important part in influencing the unconscious integration (Mudrik, Faivre, \& Koch, 2014; van Gaal et al., 2014). For instance, as mentioned above, semantic integration is more likely to occur under simultaneous than under sequential presentation. Therefore, in Experiment 1, we first presented the three priming characters of an idiom (the fourth character served as the target) simultaneously as prime. We expected to find a priming effect on the processing of the fourth target character caused by the unconscious semantic integration of the first three characters in the prime.

More importantly, we are interested in the possibility of an unconscious sequential semantic integration under the condition in which the prime characters are spaced out from left to right rather than being superimposed on one another. This methodological change will allow us to rule out one possible cause of failure to obtain semantic integration (i.e., spatial overlapping of the prime characters). In real life, our usual way of reading is to read words from left to right, including in modern Chinese, and hence presenting word sequence in the same spatial position deviates from our usual way of reading. In addition, Andersen, Essick, and Siegel (1985) found that two stimuli presented in sequence but appearing at the same spatial location could produce some neural activity, which might hamper the unconscious integration of information (Andersen et al., 1985). Also, there is evidence that presenting two stimuli in the same spatial position can cause them to be fused into a new stimulus (Eriksen, 1980; Felsten \& Wasserman, 1980). Therefore, in Experiment 2, the three priming characters of an idiom were presented sequentially, from left to right, separated by the mask, to study how this procedure affects unconscious semantic integration. In Experiment 3, we reduced the number of prime idiom characters from three to two to rule out the possibility that the failure to obtain an unconscious semantic integration in Experiment 2 is due to information overload in priming stimulus processing.

\section{Experiment 1}

In this experiment, the three priming characters of a Chinese idiom were presented simultaneously as a prime to investigate the unconscious semantic integration of idiomatic characters under the simultaneous presentation condition. The fourth character served as the target.

\section{Method}

\section{Participants}

Thirty graduate and undergraduate students from China West Normal University volunteered for this experiment. They were $18-25$ years old (mean age $=21.5$ years). All participants were right-handed, had normal or corrected-to-normal vision, and no history of (or current) neurological or psychiatric conditions. They gave their informed consent before the experiment. When asked by the experimenter, none was aware of the purpose of the experiment. After the experiment, everyone was paid for his or her participation.

\section{Stimuli and apparatus}

The refresh rate of the computer screen was $60 \mathrm{~Hz}$. Stimuli consisted of 52 frequently used Chinese four-character idioms (all the idioms and their English translations appear in the Supplementary Materials), along with the antonyms of the last character of each idiom, 26 three unrelated-character strings, and 26 capital English letters (A-Z). All of these Chinese characters were sampled from a modern Chinese dictionary. Based on the Modern Chinese Word Frequency Dictionary, the character frequency difference between the last character of idioms and their antonyms is nonsignificant, mean $=0.292$ 
vs. $0.265, t(51)=.418, p=.678$. Nor was the stroke number difference significant between the ending character of the idioms and their antonyms, $6.48 \pm .36$ (mean $\pm S E$ ) vs. $6.50 \pm$ $.34, t(51)=-.042, p=.966$. Visual stimuli were presented using E-Prime 2.0, on an ASUS 22-inch display monitor.

\section{Procedure and design}

The procedure is illustrated in Fig 1. Each trial started with a 200-ms display of a central fixation. Then, a forward mask was presented for a duration of $32 \mathrm{~ms}$. Subsequently, the first three characters of an idiom or three unrelated characters were presented simultaneously side by side, for $32 \mathrm{~ms}$. Following a 65 -ms backward mask consisting of fragmented multiple characters (see Fig. 1), an ending character or a capital letter appeared in the center of the screen until a response was made or 1,200 ms expired, whichever came first. The response was a go/no-go task in which participants pressed the " 3 " key with their index finger if they saw the ending stimulus as being a character, but refrained from responding if they saw the ending stimulus as being a letter. After the ending stimulus was cleared, the next trial began after a 1,500-ms intertrial interval. Before the experiment, participants were familiarized with the task and performed 15 practice trials.

There were three conditions varying the relationship between the prime and the ending stimulus character. In the congruent condition, the prime was the first three characters of a four-character idiom and the ending stimulus was the last character of the corresponding idiom. In the incongruent condition, the ending character was the antonym of the last character of the idiom. Both congruent and incongruent conditions required a go response. In the unrelated condition, the prime was composed of three unrelated high-frequency Chinese characters, and the final stimulus was a capital English letter. There were 52 trials in each condition. The unrelated condition served as a distractor condition, and its data were not analyzed.

Several techniques can be used to measure the visibility of the prime, such as subjective assessments, confidence judgments, postdecision wagering methods, and the forced-choice discrimination task (Overgaard, Timmermans, Sandberg, \& Cleeremans, 2010; Sandberg, Timmermans, Overgaard, \& Cleeremans, 2010; Seth, Dienes, Cleeremans, Overgaard, \& Pessoa, 2008). In the present study, we adopted the subjective assessment and forced-choice discrimination task as stimulus visibility measurement methods. For the subjective assessment, after the experiment, the experimenter asked participants to report whether or not they saw anything before the mask in order to evaluate whether they recognized the masked characters.

After the self-report survey, a forced-choice discrimination task was conducted to test the visibility of the prime stimuli. The procedure was similar to that in the main experiment, except that the ending target character was replaced with two choices, one being the first three characters of an idiom and the other being the three unrelated characters. Thus, one choice was in the prime. The participants judged which three-characters combination was presented as the prime by pressing " 1 " or " 2 " key with their right index and middle fingers, respectively. There were 104 trials for this forced discrimination task, with 52 being the three idiomatic-characters and 52 the three unrelated-characters trials. After the forced-choice discrimination task, participants were asked to report again whether or not they saw anything before the onset of the mask in the forced-choice test.

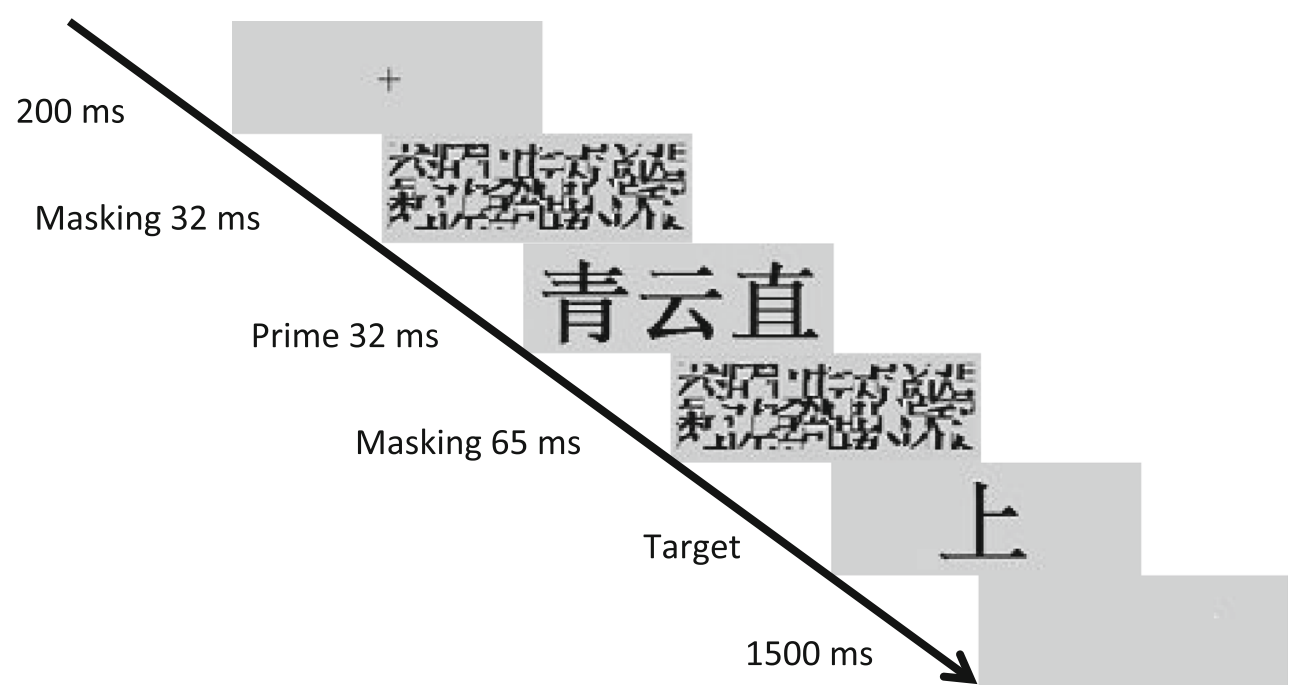

Fig. 1 Schematic of the displayed stimuli in Experiment 1 


\section{Results}

\section{Prime visibility}

Two participants reported that they could detect some Chinese characters before the mask, although they could not determine the meaning most of the time. Also, another two participants scored above chance level, with their mean percentages of correct recognition above $58 \%, p<.05$, by a binomial test. These four participants' data were excluded from further analyses. The remaining 26 participants performed at chance level in the recognition task. The mean percentage of correct recognition was $50.65 \%$, not significantly different from chance level, $t(25)=.886, p=.384$, nor was the mean $d^{\prime}$ value (mean $=0.032, S E=0.037)$ significantly different from zero, $t(25)=$ $.869, p=.393$.

\section{Overall congruency effect}

The overall accuracy of the go/no-go task was $98.42 \%$. The paired $t$ test on accuracy indicated that there was no significant difference between the congruent condition (mean $\pm S E$ : $99.31 \% \pm .48 \%)$ and the incongruent condition $(99.54 \% \pm$ $.20 \%), t(25)=-.531, p=.600$.

The paired $t$ test on RT revealed that participants responded faster in the congruent condition ( $481 \mathrm{~ms} \pm 11 \mathrm{~ms}$ ) than in the incongruent condition ( $489 \mathrm{~ms} \pm 12 \mathrm{~ms}$; see Fig. 2$), t(25)=$ $-3.128, p=.004$. Thus, an RT congruity effect was obtained from a simultaneous prime character presentation paradigm.

A separate Bayesian paired-samples $t$ test was conducted for the mean RTs using JASP. The results showed a moderate evidence for the alternative hypothesis $(\mathrm{H} 1), \mathrm{BF}_{10}=4.84$. If the hypothesis $\mathrm{H} 1$ was changed from "RTcongruent condition $\neq$ RTincongruent condition" to "RTcongruent condition < RTincongruent condition," the test result showed strong evidence for $\mathrm{H} 1, \mathrm{BF}_{10}=9.62$.

\section{Experiment 2}

In Experiment 2, the three priming characters of idioms were presented sequentially in time and from left to right in space, separated by a mask between two characters. The purpose of this experiment was to determine whether the priming effect obtained in Experiment 1 could be obtained when the prime characters were presented sequentially in time, but without superimposing characters spatially on each other.

\section{Method}

\section{Participants}

Thirty-two graduate and undergraduate students from China West Normal University volunteered for this experiment. They were $18-24$ years old (mean age $=22.6$ years). All were right-handed, had normal or corrected-to-normal vision, and no history of (or current) neurological or psychiatric conditions. They gave their informed consent before the experiment. None of the participants was aware of the purpose of the experiment. After the experiment, everyone was paid for his or her participation.

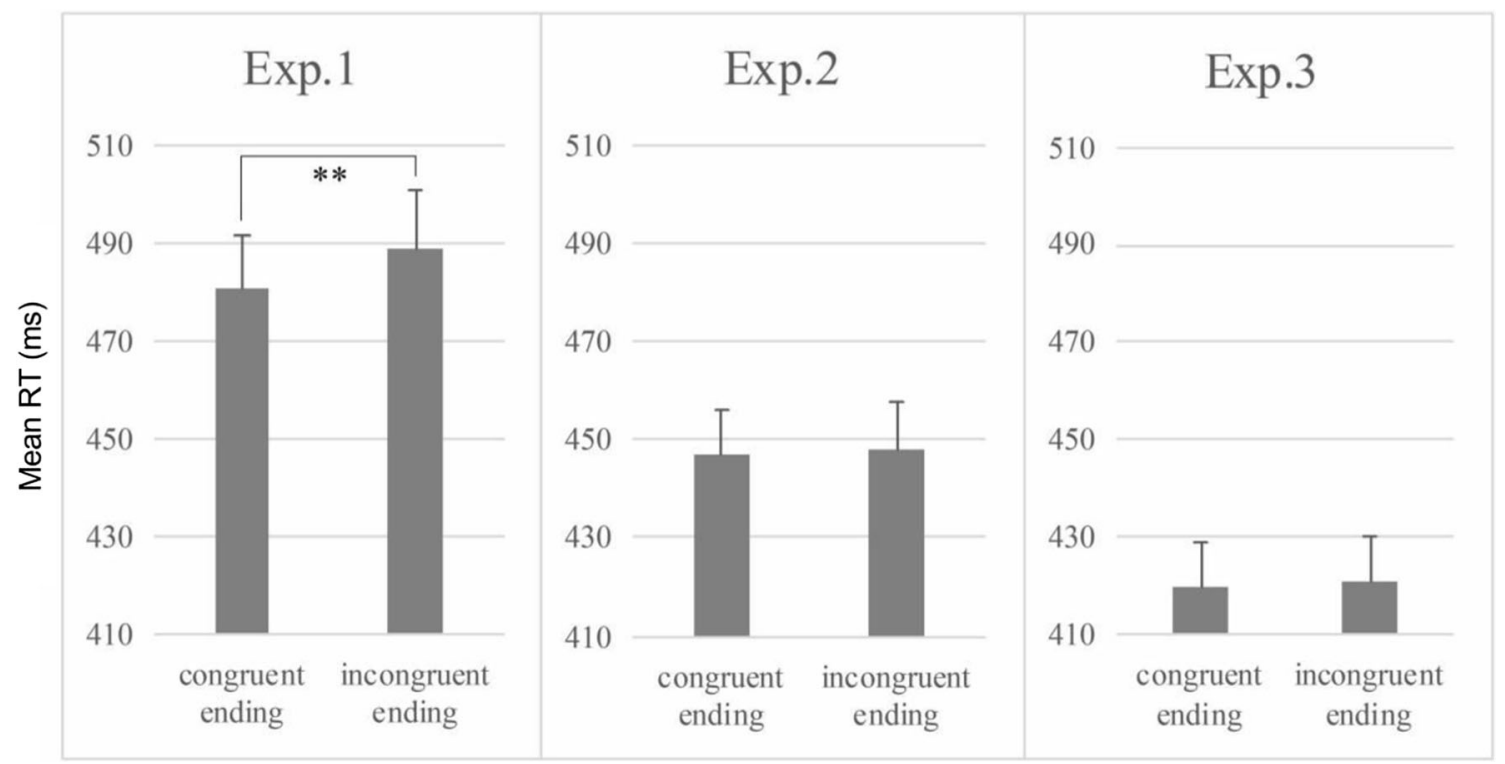

Fig. 2 Mean RTs (ms) under each prime-to-target congruence condition in the three experiments. Only in Experiment 1 did participants respond faster under the congruent condition than under the incongruent condition. There was no significant RT difference between the two conditions in Experiment 2 and 3. The error bar represents one standard error of the mean 


\section{Stimuli and apparatus}

The materials of Experiment 2 were the same as those in Experiment 1.

\section{Design and procedure}

The design and procedure of Experiment 2 were similar to those of Experiment 1 . The only difference was that the first three characters of the prime idiom in Experiment 2 were presented sequentially in time, and spatially from left to right. The left to right spatial locations on which the three characters were presented were the same as in Experiment 1. The sequence in which stimuli were presented is illustrated in Fig. 3.

As in Experiment 1, after the experiment, visibility of the prime was measured by a subjective assessment and a forcedchoice discrimination test.

\section{Results}

\section{Prime visibility}

One participant reported that she could detect some Chinese characters that appeared before the mask, although she could not determine the meaning most of the time. Also, another three participants scored above chance level, with their mean percentages of correct recognition above $58 \%, p<.05$, by a binomial test. These participants' data were excluded from further analyses. The remaining 28 participants performed at chance level in the recognition task. The mean percentage of correct recognition was $50.54 \%$, not significantly different from chance level, $t(27)=.926, p=.363$, nor was the mean $d^{\prime}$ value (mean $\left.=0.026, S E=0.028\right)$ significantly different from zero, $t(27)=.920, p=.366$.

\section{Overall congruency effect}

The overall accuracy of the go/no-go task was $98.75 \%$. The paired $t$ test revealed no significant difference in response accuracy, $(99.50 \% \pm .30 \%$ vs $99.64 \% \pm .29 \%), t(27)=$ $-.570, p=.573$, and in RT (447 ms $+9 \mathrm{~ms}$ vs. $448 \mathrm{~ms} \pm 10$ $\mathrm{ms}$ ), $t(27)=-.441, p=.663$, respectively (see Fig. 2). Thus, when a sequential presentation was used, even with the displays of the three characters spaced from left to right, no congruity effect was observed, suggesting that no semantic integration occurred in even a more favorable sequential presentation condition.

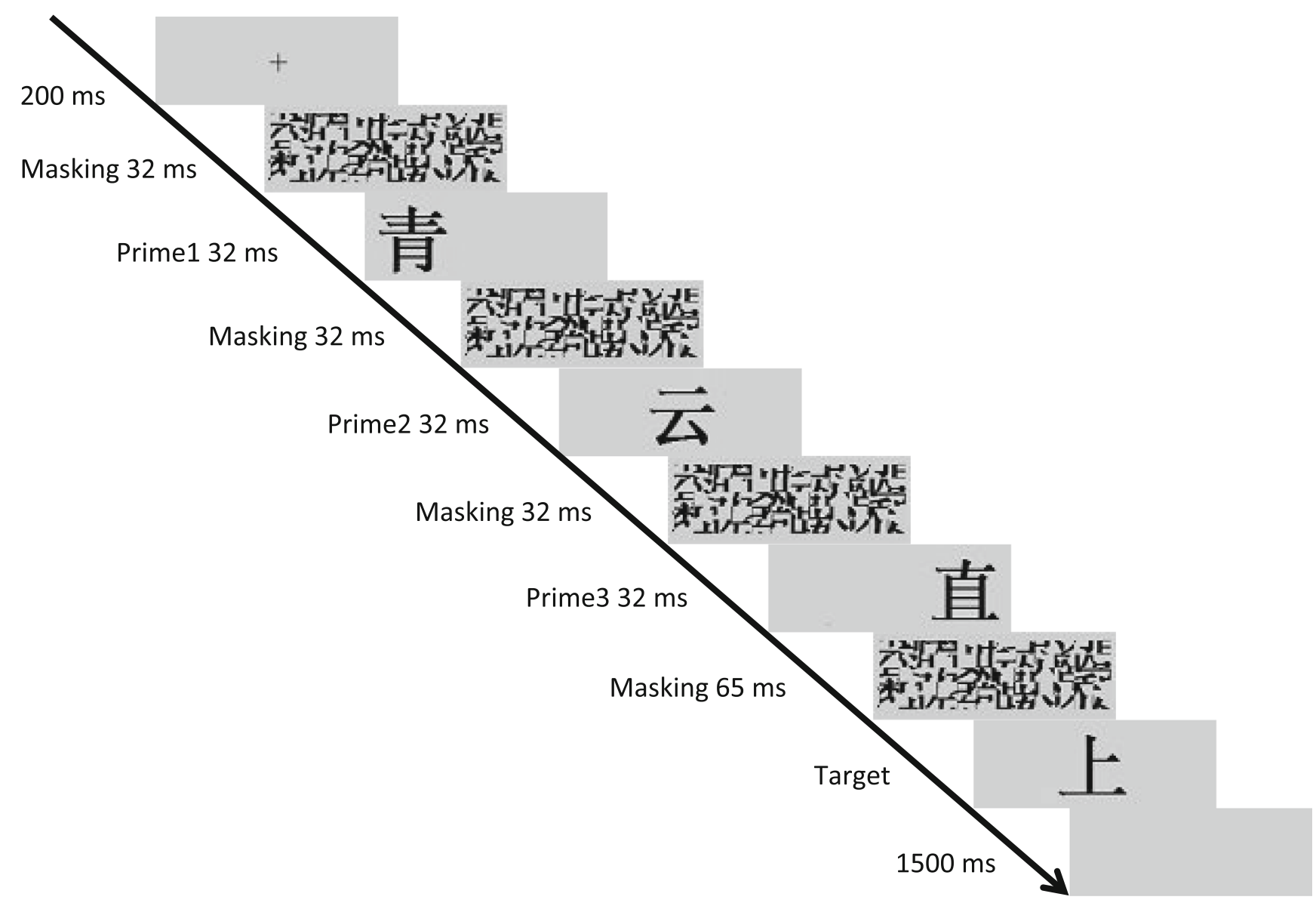

Fig. 3 Schematic of the sequentially displayed stimuli 
The results of a separate Bayesian paired-samples $t$ test for RT showed no evidence for H1 ("RTcongruent condition $\neq$ RTincongruent condition"), $\mathrm{BF}_{10}=.22$. For "RTcongruent condition < RTincongruent condition," $\mathrm{BF}_{10}=.29$.

\section{Experiment 3}

In Experiment 2, although we eliminated the prime stimulus spatial overlapping as the possible cause for the absence of a priming effect, we still did not detect a sequential integration of semantic information. The failure to obtain a congruity effect in Experiment 2 might have been caused by participants being overloaded with information at the priming stage (i.e., three characters might be too much). Therefore, in Experiment 3 , we decided to reduce the number of priming characters from three to two to lower the information processing load, whereby the sequential integration of semantic information might be easier.

\section{Method}

\section{Participants}

Twenty-eight graduate and undergraduate students from China West Normal University volunteered for this experiment. They were 19-24 years old (mean age $=21.7$ years). All were right-handed, had normal or corrected-to-normal vision, and no history of (or current) neurological or psychiatric conditions. They gave their informed consent before the experiment. None of the participants was aware of the purpose of the experiment. After the experiment, everyone was paid for his or her participation.

\section{Stimuli and apparatus}

In Experiment 3, we adopted three-character Chinese idioms (all the idioms and their English translations appear in Supplementary Materials), such as “马前卒” (literal meaning is "a foot soldier in front of a horse"; metaphoric meaning is a pawn) and “眼中钉” (literal meaning is "a nail in the eye"; metaphoric meaning is the most hated person), as our experimental materials instead of the four-character idioms. In the congruent condition, the ending target character was the third character of the idiom whose first two characters were subliminally displayed in the prime. In the incongruent condition, the ending character of one idiom was paired with the two prime characters of another idiom, such as “马前钉” and “眼中卒”, in which the first two prime characters could not be integrated with the ending target character into a meaningful idiom. However, the other properties of the ending characters, such as character frequencies and stroke numbers, were comparable in the congruent and the incongruent conditions.

\section{Design and procedure}

The design and procedure of Experiment 3 were similar to those of Experiment 2. The main difference between them was that the three prime characters in Experiment 2 were reduced to two characters, leading to a simpler type of unconscious sequential semantic integration if it could ever be achieved. Also, the ending target character in the incongruent condition was the third character of another idiom.

As in the first two experiments, after the experiments, visibility of the prime was checked out by a subjective assessment and a forced-choice discrimination task.

\section{Results}

\section{Prime visibility}

None of the participants reported that they could detect the masked characters and their meanings. However, two participants scored above chance level, with their mean percentages of correct recognition above $58 \%, p<.05$, by a binomial test. These participants' data were excluded from further analyses. The remaining 26 participants performed at chance level in the recognition task. The mean percentage of correct recognition was $50.69 \%$, not significantly different from chance level, $t(25)=.913, p=.370$, nor was the mean $d^{\prime}$ value (mean $=$ $-.103, S E=0.069)$ significantly different from zero, $t(25)=$ $-1.473, p=.153$.

\section{Overall congruency effect}

The overall accuracy of the go/no-go task was $98.81 \%$. A paired $t$ test revealed no significant differences in RT (420 $\mathrm{ms} \pm 9 \mathrm{~ms}$ vs. $421 \mathrm{~ms} \pm 9 \mathrm{~ms}), t(25)=-.170, p=$ .866 , or in accuracy of responses (100.00\% vs. $99.92 \%$ $\pm .08 \%), t(25)=1.000, p=.327$, between the congruent and the incongruent condition (see Fig. 2). Thus, we conclude that information overloading was not the cause of the failure to obtain a congruity effect in Experiment 2 .

The results of a separate Bayesian paired-samples $t$ test showed no evidence for H1 ("RTcongruent condition $\neq$ RTincongruent condition"), $\mathrm{BF}_{10}=.21$. For "RTcongruent condition < RTincongruent condition," $\mathrm{BF}_{10}=.24$.

Since post hoc participant exclusion has recently been criticized on the statistical level for issues related to the regression to the mean (Shanks, 2017), we reanalyzed the data by including the excluded participants. The results of this new analysis, presented in the Supplementary Materials section, showed that the pattern of results obtained earlier from analyses excluding fully conscious participants did not change. 


\section{General discussion}

In the current study, four-character Chinese idioms and trisyllabic (three-character) Chinese words were used to investigate whether semantic integration can occur over temporal intervals. In Experiment 1, the first three masked characters of the idioms were simultaneously presented side by side. Participants responded to the final supraliminally presented target character faster under the congruent condition than under the incongruent condition. In Experiment 2, the first three masked characters of the idioms were sequentially presented over a time period, while the display spatial locations of each character were kept the same as in Experiment 1. In Experiment 3, the number of the masked prime characters was reduced to two. However, the results of the last two experiments showed no significant congruity effect. Taken together, these results indicated that multiple masked characters can be integrated into a meaningful concept when they are simultaneously presented, but not when they are sequentially presented.

In a review paper, Mudrik, Faivre, and Koch (2014) suggested that unconscious integration was more likely to occur for a short-range than for a long-range temporal interval. The findings from the present studies that unconsciously perceived visual characters could be integrated when the characters were simultaneously presented but not when they were sequentially presented is consistent with this suggestion. Similarly, using a backward masking technique, van Gaal et al. (2014) have showed that the meanings of two successively and subliminally presented words (a modifier and an adjective — e.g., "not good") cannot be integrated into a holistic semantic unit (van Gaal et al., 2014). Likewise, Zhou et al. (2016) employed the visual crowding technique (Zhou et al., 2016) and Yang et al. (2017) used the continuous flash suppression technique (Yang et al., 2017) to explore whether the Chinese four- character idioms could be integrated without awareness. None of the results indicates that the sequentially presented characters can be integrated unconsciously. However, when semantic words were presented simultaneously, there was evidence that the masked words could be integrated without awareness (Sklar et al., 2012; van Gaal et al., 2014). The results of the three present experiments support the idea that the time interval between the characters eliminates unconscious semantic integration.

We suggest the following explanation for the different results we obtained when using simultaneous versus sequential presentations of the prime characters. In Experiment 1, the three prime characters could stimulate the cerebral cortex as a whole to match the same concept in the brain. In Experiments 2 and 3, each prime character might stimulate the cerebral cortex independently, and its effect might be fleeting, with its unconscious activation time limited to the duration of its effect (Greenwald, Draine, \& Abrams, 1996; Kiefer,
2002; Kiefer \& Brendel, 2006; Nakamura et al., 2018). In addition, the sequential integration may need the support of working memory which is primarily conscious. There is evidence that working memory is largely limited with conscious information processing (Baars \& Franklin, 2003). If there is no conscious processing, the channel through working memory may be closed, thus leading to the failure of sequential semantic integration (Zhou et al., 2016).

Perhaps another plausible explanation of our results may be the modality appropriateness hypothesis (Freides, 1974; O'Connor \& Hermelin, 1972; for a review, see Welch \& Warren, 1980). According to that idea, various sensory receptors are differentially adapted to perceiving stimuli of different modalities. Thus, vision is appropriate for the perception of spatial locations, whereas audition is most appropriate for the perception of temporally structured events. This explanation can very well account for the present results (i.e., a visual semantic integration occurring over spatial intervals, but not over temporal intervals). Humans are evolved to rely primarily on the auditory channel for communication, which requires semantic integration of sequentially presented pieces of information over temporal intervals (Hirsh, 1967). The finding that an auditory sensory register of a stimulus (the echo) persists longer than a visual sensory register (the icon) (Darwin, Turvey, \& Crowder, 1972; Sperling, 1960) is also consistent with this notion of temporally sequenced information being more suited for auditory than for visual processing (Conway, Pisoni, \& Kronenberger, 2009). Maybe this is one of the main reasons why we only observed unconscious semantic integration when the masked characters were presented simultaneously across spatial locations in Experiment 1. Future studies should use auditory materials to study the unconscious semantic integration across temporal intervals.

Mudrik et al. (2014) suggest that spatial display location may also affect the unconscious semantic integration process beyond the time integration window. In the studies that did not find unconscious sequential semantic integration, all of the masked characters/words were displayed at the same spatial location (van Gaal et al., 2014; Yang et al., 2017; Zhou et al., 2016). As noted in the introduction, sequential presentation of multiple stimuli in the same spatial position can produce neural activities that might interfere with each other and thus hamper the unconscious semantic integration (Andersen et al., 1985). In the present studies, we presented masked characters from left to right. This ruled out the above account for why semantic integration could not be obtained in a sequential presentation mode. In Experiment 3, we ruled out another possible interpretation of the failure to obtain semantic integration (i.e., information overloading). The results once again indicate that the time interval is the cause of the absence of unconscious semantic integration.

When the masked multiple characters/words are displayed simultaneously, a spatial integration is needed to achieve the 
unconscious semantic integration. When the words are presented simultaneously in a left-to-right spaced format (Sklar et al., 2012; Tu, Liu, Zhu, Jou, Zhou, \& Wan, 2019; van Gaal et al., 2014), this display format might facilitate unconscious semantic integration due to the left-to-right normal reading habit. However, when the masked multiple characters/words were displayed sequentially at the same location as in other studies (van Gaal et al., 2014; Yang et al., 2017; Zhou et al., 2016), the null results could have been caused by either a failure in sequential integration or stimulus superimposing, or by a combination of the two causes. When a standard simultaneous presentation mode (where stimuli are displayed on a line) is compared with a standard sequential presentation mode (where stimuli are displayed at the same spatial location), both the temporal and spatial factors are changed. The present studies eliminated spatial display location as a confounding factor and were able to conclude the determining factor to be the temporal variable. In Experiments 2 and 3, each prime character was fleeting, and its unconscious activation might be limited to the duration of its effect (Greenwald et al., 1996; Kiefer, 2002; Kiefer \& Brendel, 2006; Nakamura et al., 2018). The results of the three experiments together support the idea that temporal sequential integration, but not spatial integration, is the crucial factor in determining the presence or absence of semantic integration. Even though spatial display format was shown to be noncritical, it is still best for the future studies of unconscious multistimuli sequential integration to display multistimuli at different spatial locations, in order to equate that factor in the sequential display condition with that in the simultaneous display condition.

In Experiment 3, the final target character was unrelated (neutral) to the two prime characters in the incongruent condition. The difference between using an antonym and an unrelated word is that in the former case, the expectation has to be reversed (an incongruent condition), which should take more time, whereas in the latter case, the ending character was neutral with regard to the expectation which theoretically should take a response time in between the antonym (incongruent) and the fourth correct character of the idiom (congruent). That the unrelated target character did not produce an RT longer than that in the congruent condition in Experiment 3 suggests that the unrelated (neutral) target character did not make the condition more difficult than the antonym condition in Experiment 2.

Considering the combined findings of the present studies and other studies of unconscious sequential integration using low-level materials, such as symbols (Atas, Faivre, Timmermans, Cleeremans, \& Kouider, 2013) and arrows (Tu, Zhu et al., 2019), it is possible that such factors as time, space, and processing level (Faivre, Mudrik, Schwartz, \& Koch, 2014) all influence the unconscious integration. Furthermore, this influence might not follow the all-or-none law, but rather may be continuous in nature. Indeed, the concept of consciousness as a continuous construct is supported in the quantitative measure of the level of consciousness with EEG (Casali et al., 2013), in partial awareness hypothesis (Kouider, de Gardelle, Sackur, \& Dupoux, 2010), and in the interaction between top-down and bottom-up processing (Tu et al., 2015).

Finally, post hoc participant exclusion widely used in studies on unconscious information processing has recently been criticized on the statistical level for issues related to the regression to the mean (Shanks, 2017). The essence of the idea of regression toward the mean is that when two variables are not perfectly correlated (such as the awareness measure and the conscious performance measure), the participants who measure lower in one variable tend to measure higher in the other. As such, participants who measure lower in awareness tend to measure higher in unconscious task performance and vice versa, thus producing spurious evidence of unconscious information processing if the aware participants are excluded. To address this issue, we reanalyzed the data by including the excluded participants. The results of this new analysis, presented in the Supplementary Materials section, show that the pattern obtained earlier from the original analyses did not change. Although the exclusion rate (i.e., very low) and criteria used in this study may be different from those cases considered in Shanks' (2017) article, we remind the readers that they should not interpret unconscious processing in this article too strictly, due to the inclusion of a small number of aware participants.

Acknowledgements We thank Richard Harris for editing the language of the final draft of this paper and giving valuable comments. This work was supported by a funding for the Start-up Project of Scientific Research on Introducing Talents to Guizhou University of Finance and Economics in 2018 (Grant No. 2019YJ020) and from the National Natural Science Foundation of China (Grant No. 31500871).

Open practices statements None of the data or materials for the experiments reported here are available, and none of the experiments were preregistered.

\section{References}

Andersen, R. A., Essick, G. K., \& Siegel, R. M. (1985). Encoding of spatial location by posterior parietal neurons. Science, 230(4724), 456-458.

Atas, A., Faivre, N., Timmermans, B., Cleeremans, A., \& Kouider, S. (2013). Nonconscious learning from crowded sequences. Psychological Science, 25(1), 113-119.

Baars, B. J., \& Franklin, S. (2003). How conscious experience and working memory interact. Trends in Cognitive Sciences, 7(4), 166-172.

Casali, A. G., Gosseries, O., Rosanova, M., Boly, M., Sarasso, S., Casali, K. R., . . . Massimini, M. (2013). A theoretically based index of consciousness independent of sensory processing and behavior. Science Translational Medicine, 5, 198ra105. doi:https://doi.org/ 10.1126/scitranslmed.3006294 
Conway, C. M., Pisoni, D. B., \& Kronenberger, W. G. (2009). The importance of sound for cognitive sequencing abilities. Current Directions in Psychological Science, 18, 275-279.

Darwin, C. J., Turvey, M. T., \& Crowder, R. G. (1972). An auditory analog of the Sperling partial report procedure: Evidence for brief auditory storage. Cognitive Psychology, 3, 255-267.

Eriksen, C. W. (1980). The use of a visual mask may seriously confound your experiment. Perception \& Psychophysics, 28(1), 89-92.

Faivre, N., Mudrik, L., Schwartz, N., \& Koch, C. (2014). Multisensory integration in complete unawareness. Psychological Science, 25(11), 2006-2016.

Felsten, G., \& Wasserman, G. S. (1980). Visual masking: Mechanisms and theories. Psychological Bulletin, 88(2), 329-353.

Freides, D. (1974). Human information processing and sensory modality: Cross-modal functions, information complexity, memory, and deficit. Psychological Bulletin, 81, 284-310.

Greenwald, A. G., Draine, S. C., \& Abrams, R. L. (1996). Three cognitive markers of unconscious semantic activation. Science, 273(5282), $1699-1702$.

Hirsh, I. J. (1967). Information processing in input channels for speech and language: The significance of serial order of stimuli. In F. L. Darley (Ed.), Brain mechanisms underlying speech and language (pp. 21-38). New York, NY: Grune \& Stratton.

Kiefer, M. (2002). The N400 is modulated by unconsciously perceived masked words: Further evidence for an automatic spreading activation account of N400 priming effects. Cognitive Brain Research, 13(1), 27-39.

Kiefer, M., \& Brendel, D. (2006). Attentional modulation of unconscious "automatic" processes: Evidence from event-related potentials in a masked priming paradigm. Journal of Cognitive Neuroscience, 18(2), 184-198.

Kouider, S., de Gardelle, V., Sackur, J., \& Dupoux, E. (2010). How rich is consciousness? The partial awareness hypothesis. Trends in Cognitive Sciences, 14(7), 301-307.

Mudrik, L., Faivre, N., \& Koch, C. (2014). Information integration without awareness. Trends in Cognitive Sciences, 18(9), 488-496.

Nakamura, K., Makuuchi, M., Oga, T., Mizuochiendo, T., Iwabuchi, T., Nakajima, Y., \& Dehaene, S. (2018). Neural capacity limits during unconscious semantic processing. European Journal of Neuroscience, 47(8), 929-937.

O'Connor, N., \& Hermelin, B. (1972). Seeing and hearing in space and time. Perception \& Psychophysics, 11, 46-48.

Overgaard, M., Timmermans, B., Sandberg, K., \& Cleeremans, A. (2010). Optimizing subjective measures of consciousness. Consciousness and Cognition, 19(2), 682-684.

Sandberg, K., Timmermans, B., Overgaard, M., \& Cleeremans, A. (2010). Measuring consciousness: Is one measure better than the other? Consciousness and Cognition, 19(4), 1069-1078.
Seth, A. K., Dienes, Z., Cleeremans, A., Overgaard, M., \& Pessoa, L. (2008). Measuring consciousness: Relating behavioural and neurophysiological approaches. Trends in Cognitive Sciences, 12(8), 314 321.

Shanks, D. R. (2017). Regressive research: The pitfalls of post hoc data selection in the study of unconscious mental processes. Psychonomic Bulletin \& Review, 24(3), 752-775.

Sklar, A. Y., Levy, N., Goldstein, A., Mandel, R., Maril, A., \& Hassin, R. R. (2012). Reading and doing arithmetic nonconsciously. Proceedings of the National Academy of Sciences, 109(48), 19614-19619.

Sperling, G. (1960). The information available in brief visual presentations. Psychological Monographs: General and Applied, 74(11), 1 29.

Tu, S., Jou, J., Cui, Q., Zhao, G., Wang, K., Hitchman, G., . . Zhang, Q. (2015). Category-selective attention interacts with partial awareness processes in a continuous manner: An fMRI study. Cogent Psychology, 2(1), 1046243. doi:https://doi.org/10.1080/23311908. 2015.1046243

Tu, S., Liu, C., Zhu, S., Jou, J., Zhou, Y., \& Wan, S. (2019). The semantic integration between two subliminally perceived words simultaneously presented at different locations. Journal of Psycholinguistic Research. Advance online publication. doi:https:// doi.org/10.1007/s10936-019-09648-9

Tu, S., Zhu, S., Liang, Q., Jou, J., Wan, S., Zhao, G., . . Jiang, Q. (2019). Unconscious integration of sequentially presented subliminal arrow pointing directions. Australian Journal of Psychology. Advance online publication. doi: https://doi.org/10.1111/ajpy.12252

van Gaal, S., Naccache, L., Meuwese, J. D., van Loon, A. M., Leighton, A. H., Cohen, L., \& Dehaene, S. (2014). Can the meaning of multiple words be integrated unconsciously? Philosophical Transactions of the Royal Society London B Biological Science, 369(1641). doi:https://doi.org/10.1098/rstb.2013.0212

Welch, R. B., \& Warren, D. H. (1980). Immediate perceptual response to intersensory discrepancy. Psychological Bulletin, 88(3), 638-667.

Yang, Y., Tien, Y., Yang, P., \& Yeh, S. (2017). Role of consciousness in temporal integration of semantic information. Cognitive, Affective, \& Behavioral Neuroscience, 17(5), 954-972.

Zhou, J., Lee, C., Li, K., Tien, Y., \& Yeh, S. (2016). Does temporal integration occur for unrecognizable words in visual crowding? PLOS ONE, 11(2), e149355.

Publisher's note Springer Nature remains neutral with regard to jurisdictional claims in published maps and institutional affiliations. 